\title{
Magnetoelastic Coupling in Ni-Mn-Ga Magnetic Shape Memory Alloy
}

\author{
Oleg Heczko \\ Institute of Physics of the AS CR, Na Slovance 2, 18221 Praha 8, Czech Republic \\ heczko@fzu.cz
}

Keywords: magnetically induced reorientation (MIR), magnetic shape memory effect, ordinary magnetostriction, magnetoelastic coupling, Ni-Mn-Ga.

\begin{abstract}
The role of magnetoelastic coupling in the mechanism of magnetically induced reorientation or redistribution (MIR) of twin variants is still a matter of some controversy. To evaluate this role ordinary magnetostriction of different $\mathrm{Ni}-\mathrm{Mn}-\mathrm{Ga}$ single crystals transforming to 5M (exhibiting MIR) and NM (no MIR) martensite were measured. The magnetostriction of Ni-MnGa austenite is relatively low and steeply increases when approaching to martensite transformation. This is correlated to the softening of elastic modulus. Observed high field contribution of opposite sign may be due to the dependence of higher order elastic constant on magnetic field. The magnetostriction of martensite is difficult to determine as it is masked by much stronger MIR effect and indirect method must be used. The results are discussed in the frame of magnetoelastic model for MIR and compared with magnetic energy model.
\end{abstract}

\section{Introduction}

Magnetic shape memory effect includes two different phenomena, magnetically induced transformation in Ni-Mn-Ga from austenite to martensite (MIM) and magnetically induced reorientation or redistribution (MIR) of twin martensitic variants without structural changes [1]. The role of usual magnetostriction, i.e. a deformation of crystal lattice due to the rotation of magnetic moment, is a matter of controversy. Here we will consider the role of magnetostriction in MIR. Model of Lvov and Chernenko [2,3] based on Landau theory of phase transformation [4] considers the magnetoelastic interaction crucial for twin boundary motion in magnetic field and thus for the existence of MIR. Surprisingly there is only limited knowledge about the magnetostriction even in the most investigated MSM material as Ni-Mn-Ga [1,5,6]. The magnetostriction of Ni-Mn-Ga austenite increases when approaching to martensite transformation [5]. The magnetostriction of martensite is difficult to determine as it is masked by much stronger MIR effect and indirect methods must be used [6].

To evaluate the role of ordinary magnetostriction in MIR the magnetostriction of different NiMn-Ga alloys transforming to 5M martensite (exhibiting MIR) and NM martensite (no MIR) were measured. Obtained results are discussed in the frame of magnetoelastic model and compared with other models for twin boundary motion in magnetic field.

\section{Experimental}

Single crystalline materials of $\mathrm{Ni}_{49.6} \mathrm{Mn}_{29.2} \mathrm{Ga}_{21.2}$ with $5 \mathrm{M}$ structure (also designated as 10M) [7] and $\mathrm{Ni}_{56.9} \mathrm{Mn}_{18} \mathrm{Ga}_{25.1}$ of NM structure were used. The shape of samples was planparallel prism with the faces of approximately $\{100\}$ planes of parent austenite. The compression was used to prepare single variant martensite sample. The transformation temperatures were determined by magnetic susceptibility [8] and by DSC (above Curie point in NM case). Both samples were in martensite state at room temperature. The change of dimension with temperature and in magnetic field at constant temperature was measured by capacity dilatometer inserted in Quantum Design PPMS. Maximum field used was 2T. The direction of field was perpendicular to the direction of measured dilatation which was also the easy axis of magnetization $<001>$ in martensitic state. The samples with easy axis were prepared by compression. 


\section{Results and discussion}

The magnetostriction occurs from rotation of magnetization from the easy axis to the field direction and originates mostly from the spin orbit coupling. The coupling between magnetization and elastic properties in certain direction can be described by magnetoelastic coupling coefficients

$$
\mathrm{B}_{\mathrm{ij}} \propto \mathrm{c}_{\mathrm{ijkl}} \lambda_{\mathrm{kl}}
$$

where $\mathrm{c}_{\mathrm{ijkl}}$ is elastic constants and $\lambda_{\mathrm{kl}}$ is measured strain in magnetic field. In [100] direction magnetostriction is $\lambda_{100} \approx \mathrm{B}_{1} /\left(\mathrm{c}_{11}-\mathrm{c}_{12}\right)$. The elastic constant difference for isotropic material can be written as $\mathrm{c}_{11}-\mathrm{c}_{12}=\mathrm{E}(1-\mathrm{v}) /((1+\mathrm{v}) *(1-2 v))-\mathrm{Ev} /((1+v) *(1-2 v))=\mathrm{E} /(1+v)$, where $\mathrm{E}$ is Young modulus and $v$ is Poisson number [9]. In approximation for cubic in [100] orientation and by further simplification we thus get

$$
\lambda \approx \mathrm{B} / \mathrm{E}
$$

where $\mathrm{B}$ can be considered as a stress causing given magnetostriction.

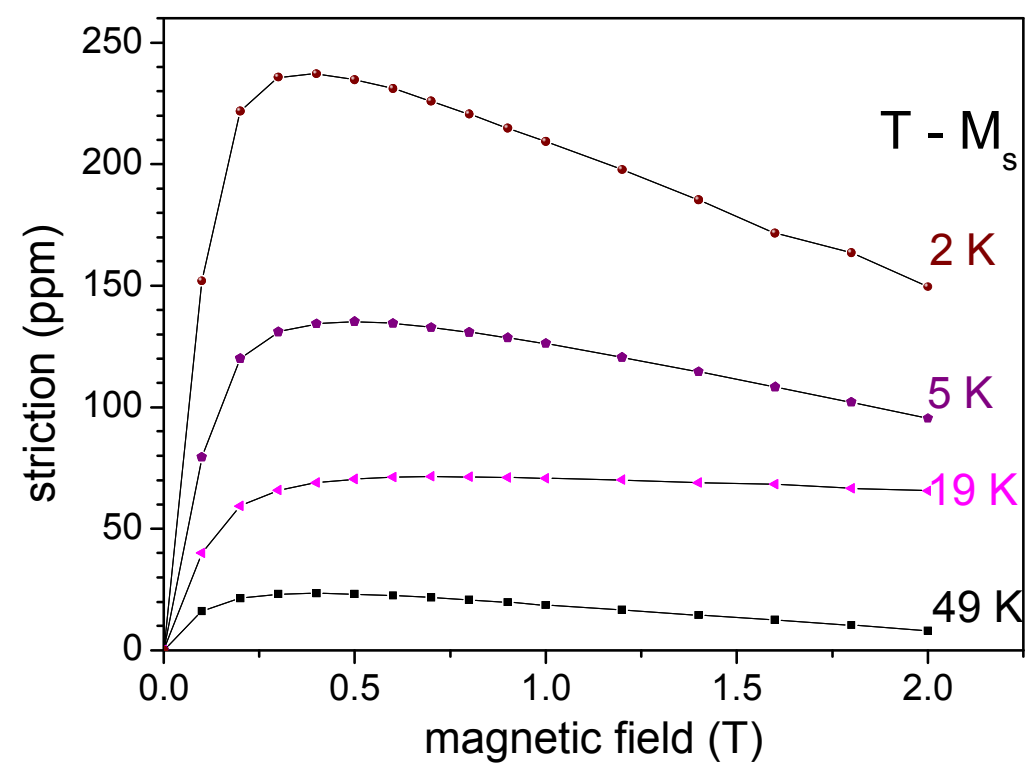

Fig. 1: Dilatation perpendicular to magnetic field at different temperatures. In figure the difference between martensite start and measurement temperatures is marked.

Magnetostriction in austenite was measured during cooling. Fig. 1 shows selected curves of fieldinduced strain. Initial increase of the deformation is due to magnetization rotation (usual, ordinary magnetostriction). In the maximum of the curve (about 0.25T) the sample is saturated, magnetization is along field and no further magnetization rotation and thus magnetostriction occurs. Therefore the origin of observed decrease in higher field must be different. In this region magnetic moment is saturated and only Zeeman energy increases with increasing field. This can lead to change of the elastic properties of the material and consequent lattice stiffening. However, to explain the observed decrease we cannot exclude a form effect due to magnetostatic energy in short sample [10] or some measurement artifacts as slight rotation of the sample having slightly nonparallel sides. More detailed measurements are planned.

Fig. 2 summarizes the temperature dependence of field-induced strain. The measured strain increases sharply in the vicinity of martensitic transformation. The increase of the magnetostriction is similar as shown by Tickle and James [5] and Kokorin and Wuttig [11]. It was proposed that the 
increase is due to lattice softening and consequent change of elastic constants. In further simplification for the approximation of elastic properties we will use a temperature dependence of storage modulus $\mathrm{E}(\mathrm{T})$ measured by dynamic mechanical analyzer (DMA) presented in [12]. According Eq. 3 the magnetostriction $\lambda$ is inversely proportional to the modulus E. From this relation we can estimate the value of the magnetoelastic coupling in the austenite.

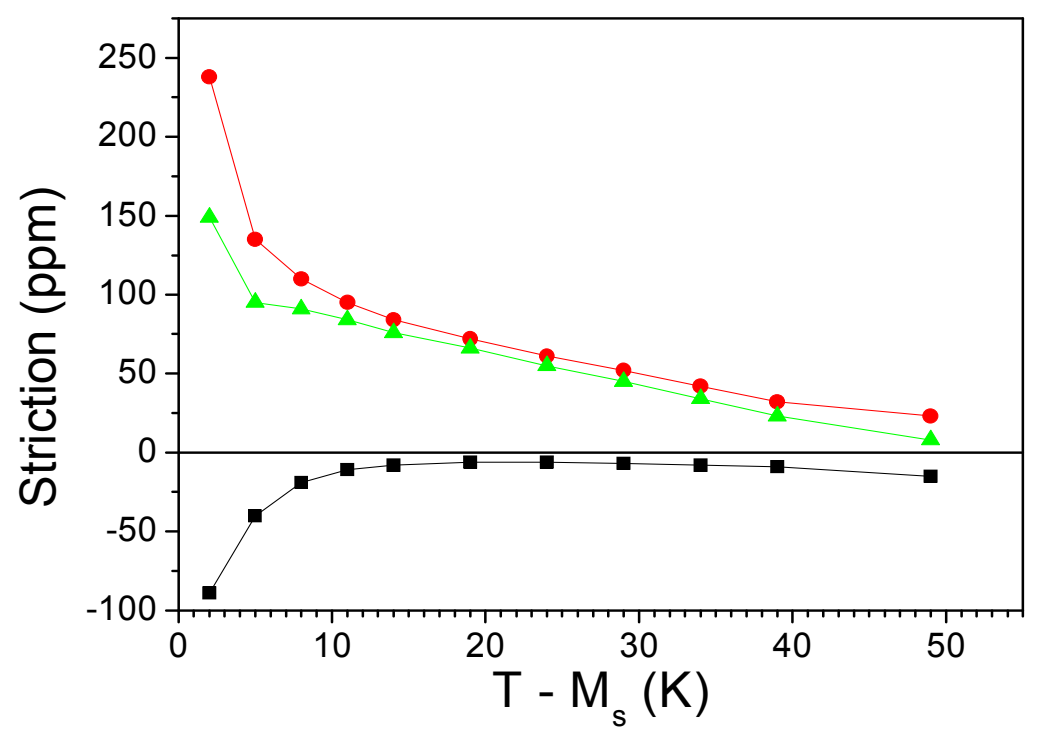

Fig. 2: Temperature dependence of maximum measured strain in the field up to $2 \mathrm{~T}$ (see Fig. 1) as a function of temperature interval from martensite start temperature $\mathrm{M}_{\mathrm{s}}=303 \mathrm{~K}$. Triangle (green) total deformation in the field; circle (red) - magnetostriction; square (black) - high field strain contribution.

In Fig. 3 there is the comparison of measured magnetostriction and the strain obtained using Eq. 3 and suitable proportional constant. From this proportional constants we can roughly approximate the magnetoelastic coupling constant $\mathrm{B}=0.8 \mathrm{MPa}$ assuming that the magnetostriction in field direction is twice the value measured perpendicularly. This constant is about ten times less than for pure Ni [9].

To measure magnetostriction in $5 \mathrm{M}$ martensite the same method fails as small magnetostrictive strain is swamped by large strain due to MIR. It can be measured in stress-constrained sample, which suppresses MIR [13] or indirectly as demonstrated in [6]. It was estimated there that the magnetostriction in $5 \mathrm{M}$ is less that $100 \mathrm{ppm}$ at room temperature. This value is consistent with the value estimated from elastic modulus (see Fig. 3), which on turn would suggest similar magnetoelastic coupling in austenite and martensite. However, the measured elastic modulus of martensite can be affected by twin boundary motion [14].

Alternatively we can determine the magnetostriction in NM martensite, in which twin boundaries are immobile and thus ultimately constrained. Measured magnetostriction was of order $30 \mathrm{ppm}$ and nearly constant over large temperature interval to the vicinity of Curie temperature. Although it is even smaller value than determined value for $5 \mathrm{M}$, estimated magnetoelastic coupling is stronger as the elastic modulus is much higher than in modulated phases [12]. Of course one might questioned the relevance of determined magnetostriction in NM for other kinds of martensite. But one could assume that the magnetoelastic coupling cannot differ too much as the magnetic properties and composition does not differ very much. Moreover, there is suggestion that modulated martensites might be a special case of adaptive phase consisting of nanotwinned NM martensite [15] and thus the properties of modulated phases are some kind of average of the NM martensite properties. 


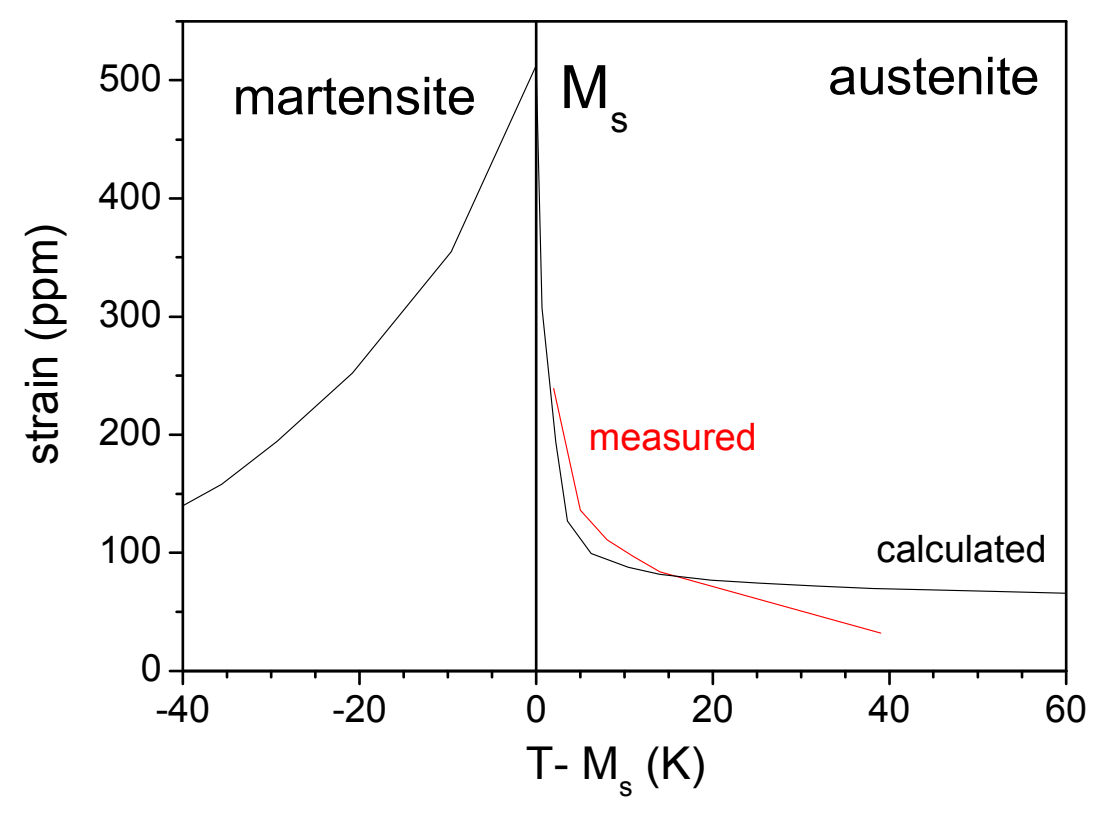

Fig. 3: Measured and calculated strain (as marked in the figure). Calculated strain was obtained from the elastic modulus E using proportional constant to fit the measured strain.

Measured magnetostriction in martensite can be used to evaluate different model for the twin boundary motion in magnetic field. At first we have to consider field dependence of magnetization. In used rectangular specimen with in homogenous distribution of internal field and complex magnetic domain configurations, it is very difficult or nearly impossible to calculate the field dependence of magnetostriction. However, if we approximate magnetization process by magnetization rotation, which is valid in material with strong uniaxial anisotropy magnetized perpendicularly to easy axis, the magnetization is linear function of magnetic field. This ideal magnetization curve is shown in Fig. 4. For magnetostriction in this case we can write $\lambda \propto\left(M / M_{\mathrm{s}}\right)^{2}$, where $\mathrm{M}$ is field dependent magnetization and $\mathrm{M}_{\mathrm{s}}$ is saturation magnetization.

In magnetoelastic model of twin boundary motion, equivalent stress can be approximate as

$$
\sigma_{\lambda} \approx \mathrm{E} \lambda \approx \mathrm{E}\left(M / M_{\mathrm{s}}\right)^{2}
$$

thus it depends on square of magnetic field. This dependence is charted in Fig. 4. Maximum stress is then given by saturation magnetostriction, $\lambda_{\mathrm{s}}$.

In other model proposed by Likhachev and Ullakko [16] we consider an equivalent magnetic stress, $\sigma_{\text {mag, }}$ obtained from the difference of magnetic energy between variants, $\Delta E_{\text {mag. For }}$ simplified structure of two variants with easy and hard axis along the field we get $[17,18]$

$$
\sigma_{\mathrm{mag}}(H)=\Delta E_{\mathrm{mag}} / \varepsilon_{0}=\left(M_{\mathrm{s}} H-1 / 2 M_{\mathrm{s}} H^{2} / H_{\mathrm{a}}\right) / \varepsilon_{0},
$$

where $\varepsilon_{0}$ is tetragonal distortion. Here we are using ideal magnetization curves without hysteresis and neglecting a demagnetization [9]. Maximum stress is then given by magnetic anisotropy, $\mathrm{K}_{\mathrm{u}}$. It is apparent from Fig. 4, there is not very large difference between models and moreover, the strong increase of the magnetic stress in low field is flattened if we consider an effect of demagnetization as shown in [17]. Nonetheless, the difference particularly in low field region may provide means to test the models as the difference between them is the highest compared to absolute magnitude of the stress. Particularly for the materials which exhibit the twinning stress less than $0.5 \mathrm{MPa}$ and reorientation occurs in the field lower than $0.1 \mathrm{~T}$ [19], i.e. about $0.2 \mathrm{H} / \mathrm{H}_{\mathrm{a}}$ the difference should be measurable. It seems that indeed there is correlation between twinning stress and field, which would 
point to magnetic model [20]. Additionally to obtain the same level of equivalent stress the magnetoelastic coupling has to be stronger than measured.

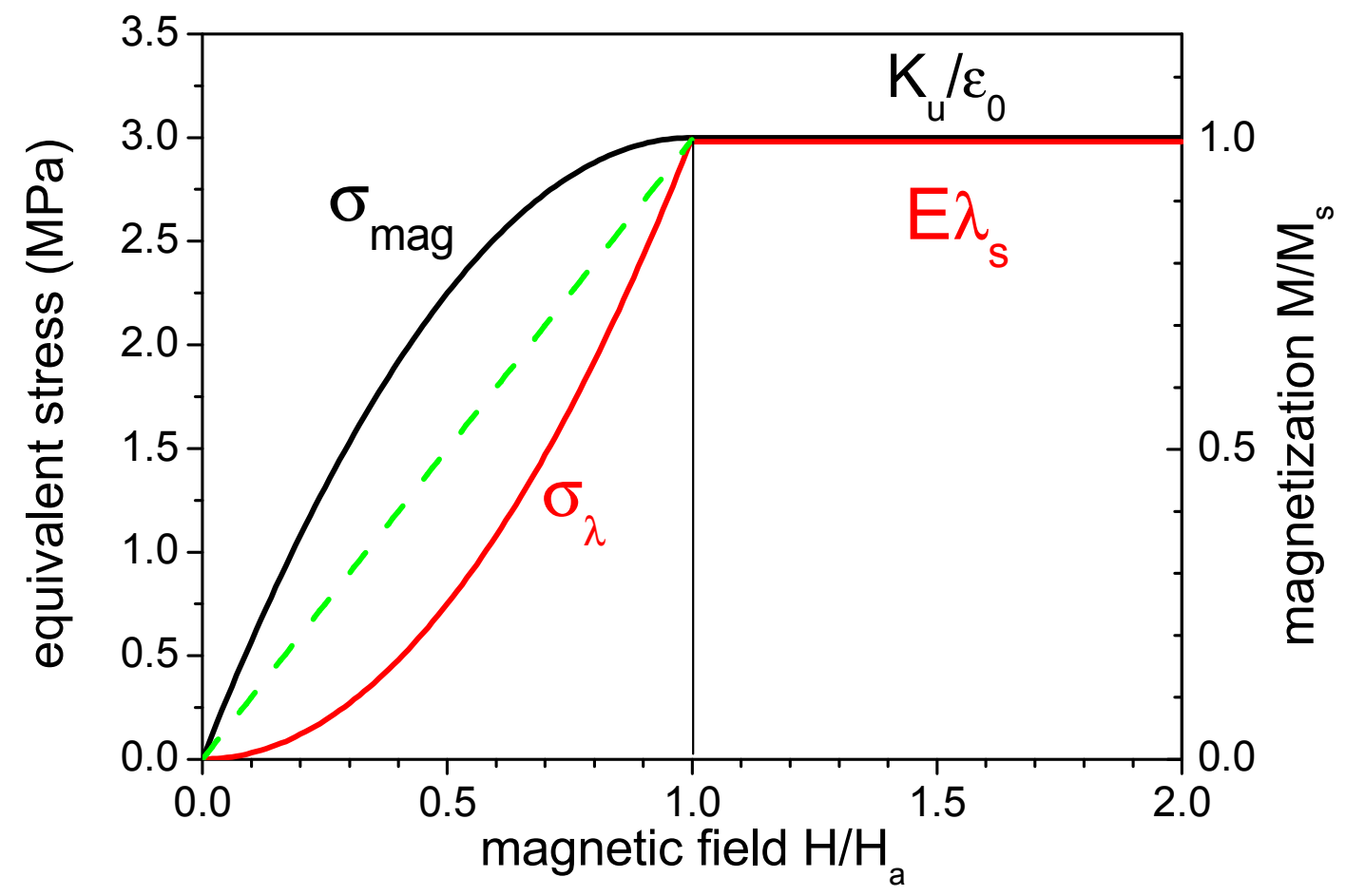

Fig. 4: Ideal magnetization curve for uniaxial material magnetized perpendicularly to easy axis

(magnetization rotation) - green dash line. Field dependences of equivalent stress calculated according models marked in figure; black - model of magnetic stress $\left(\mathrm{K}_{\mathrm{u}}=1.8 \times 10^{5} \mathrm{~J} / \mathrm{m}^{3}, \varepsilon_{0}=6 \%\right)$; red - magnetoelastic model $\left(\mathrm{E}=10 \mathrm{GPa}, \lambda_{\mathrm{s}}=300 \mathrm{ppm}\right)$.

\section{References}

[1] K. Ullakko, et al.: Appl. Phys. Lett. 69, (1996) p.1966

[2] V.A. Chernenko, et al.: Phys Rev. B 69, (2004) 134410

[3] V. A. L’vov, S.P. Zagorodyuk, and V. A. Chernenko: Eur.Phys. J. B Vol. 27 (2002), p. 55

[4] V. A. L'vov, E. V. Gomonaj, and V. A. Chernenko: J. Phys.: Condens. Matt. 10, (1998) p. 4587

[5] R. Tickle, R.D. James: J. Magn. Magn. Mat. 195 (1999) p. 627

[6] O. Heczko, J. Magn. Magn. Mat. 290-291, (2005) p. 846

[7] Y. Ge et al.: J. Phys. IV 112, (2003), p. 921

[8] O. Heczko et al.: J. Magn. Magn. Mat. Vol. 242-245 (2002) p. 1446

[9] O'Handley: Ch. 7 Magnetoelastic effects in Modern magnetic materials, John Wiley \& Sons, Inc, (2000)

[10] D.B. Cullity: Introduction to Magnetic Materials, Addison-Wesley Publishing Company, Reading, Massachusetts, (1972), p. 266.

[11] V.V. Kokorin, M. Wuttig, J. Magn.Magn. Mat. 234 (2001) p. 25.

[12] I. Aaltio et al.: Mater. Sci. Eng. A 481-482 (2008) p. 314.

[13] O. Heczko, et al: Mater. Sci. Forum Vol. 373-3 (2001), p. 341 
[14] O. Soderberg et al.: Mater. Sci. Eng. A, Vol. 386 (2004), p. 27

[15] S. Kaufman et al.: Adaptive martensite, submitted (2009) arxiv.org/pdf/0906.5365

[16] A.A. Likhachev, and K. Ullakko, Phys. Lett. A275, (2000), p. 142.

[17] L. Straka, O. Heczko: IEEE Trans Mag, 39, (2003), p. 3404

[18] L. Straka, O. Heczko: J. Magn. Magn. Mat. Vol.290 (2005) p. 829

[19] O. Heczko, A. Soroka, S.-P. Hannula: Appl. Phys. Lett., Vol. 93 (2008), 022503

[20] I. Aaltio, unpublished results, private communication. 